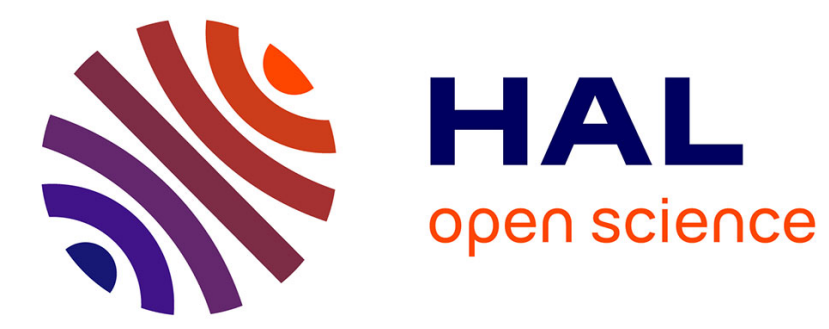

\title{
Fracture reveals clustering in cohesive granular matter
}

Franco Tapia, Stéphane Santucci, Jean-Christophe Géminard

\section{To cite this version:}

Franco Tapia, Stéphane Santucci, Jean-Christophe Géminard. Fracture reveals clustering in cohesive granular matter. EPL - Europhysics Letters, 2016, 115 (6), pp.64001. 10.1209/0295-5075/115/64001 . hal-01417941

\section{HAL Id: hal-01417941 \\ https://hal.science/hal-01417941}

Submitted on 21 Dec 2016

HAL is a multi-disciplinary open access archive for the deposit and dissemination of scientific research documents, whether they are published or not. The documents may come from teaching and research institutions in France or abroad, or from public or private research centers.
L'archive ouverte pluridisciplinaire HAL, est destinée au dépôt et à la diffusion de documents scientifiques de niveau recherche, publiés ou non, émanant des établissements d'enseignement et de recherche français ou étrangers, des laboratoires publics ou privés. 


\title{
Fracture reveals clustering in cohesive granular matter
}

\author{
Franco Tapia, Stéphane Santucci and Jean-Christophe GÉminard \\ Univ Lyon, Ens de Lyon, Univ Claude Bernard, CNRS, Laboratoire de Physique, F-69342 Lyon, France.
}

\author{
PACS 45.70.-n-Granular systems \\ PACS 47.57.Gc - Granular flow \\ PACS $62.20 . \mathrm{mm}$ - Fracture
}

\begin{abstract}
We report an experimental study of the morphology of fractures in cohesive granular materials. Cohesion is introduced by equilibrating the grains with a humid atmosphere. The setup allows to produce a controlled crack in a thin layer of a glass beads assembly, and observe with an extremely high resolution the edge of the fracture at the free surface of the layer. The detailed multi-scale analysis of the fracture profile reveals the presence, in the bulk of the material, of clusters of grains whose size increases monotonically with the relative humidity. These results are important because the formation of clusters, resulting in a heterogeneity of the cohesion force, governs the mechanical properties of cohesive granular matter in contact with a humid atmosphere.
\end{abstract}

Introduction. - It is of common knowledge that humidity can alter the physical properties of granular matter. The first basic proof is the possibility to build a castle with wet sand, whereas dry sand will only flow $[1,2]$. When granular materials are placed in a humid atmosphere, their properties slowly evolve with time due to the formation of liquid bridges that progressively bond the grains together. Such process leads for instance to an increase of the angle of avalanche [3] or of the friction coefficient [4].

Cohesive granular materials fracture rather than flow [5]. Indeed, when a thin layer of cohesive grains is deformed, one can observe the formation of a regular pattern at the free surface of the layer [6]. Its characteristic size, $\mathcal{L}$, is not only proportional to the layer thickness, but also depends on the grain size and relative humidity in a peculiar way. More precisely, even if a decrease in the grain size and an increase of the relative humidity both enhance the cohesion, $\mathcal{L}$ does not depend on the grain size at a given relative humidity whereas $\mathcal{L}$ increases drastically with the relative humidity for a given grain size. Alarcón et al [7] suggested that such experimental observations could only be accounted for by the formation of cohesive clusters in the bulk of the material.

Nevertheless, the direct observation of cohesive clusters in the bulk of a granular material is a difficult task. Authors reported the formation of clusters when liquid is introduced in the system [8]. In this case, fully developped liquid bridges form at the contact points between the grains. Their size compares to the grain size and they can be directly, even if not easily, observed. However, when the granular material is placed in contact with a humid atmosphere, the liquid content is much smaller, leading to the formation of bridges at the scale of the asperities present at the grain surface. To our knowledge, the bridges cannot be observe in this case and the formation of clusters can be revealed indirectly, only. For instance, some experiments revealed that, during the discharge of a silo, flows of cohesive granular material involve groups of grains rather than grains alone [5]. Therefore, there is a need for experiments providing more clues about the distribution of the liquid and the heterogeneity of the cohesion in order to understand the mechanics of cohesive granular matter. In the present article, we propose to observe and characterize the profile of a fracture, produced in a thin layer of cohesive granular material. Assuming that the fracture propagates along the weakest path in the material, thus around the clusters, we propose to get their typical size from the geometrical characteristics of the fracture.

Experimental setup and protocol. - The experiment consists in breaking a layer of cohesive granular material along a well-defined, in-plane, direction. A thin layer of cohesive granular material is deposited upon a flat and horizontal surface constituted of two acrylic plates in contact through one of their rectilinear edges (Fig. 1, bottom). A, nominally rectilinear, fracture is produced by gently separating the two plates.

In practice, the two plates $\left(4 \times 20 \mathrm{~cm}^{2}\right)$ rest onto a flat acrylic table $\left(10 \times 20 \mathrm{~cm}^{2}\right)$, which insures that they are displaced only in the horizontal plane when separated. 
This set-up is placed inside a transparent acrylic chamber with a top glass window, which permits the control of the ambient humidity and temperature. The temperature, which is measured but not actively controlled, remains in all the experiments about $T=22{ }^{\circ} \mathrm{C}$. The humidity is tuned by equilibrating the air in the chamber with saturated salt solutions [9]. The resulting relative humidity at equilibrium, $R_{H}$, is measured thanks to a PID-controller (FOX-301A with a humidity/temperature sensor DS4000/NTC) which also provides a measure of the temperature, $T$. We report experiments for $R_{H}=$ $33,57,75,88$, and $100 \%$.

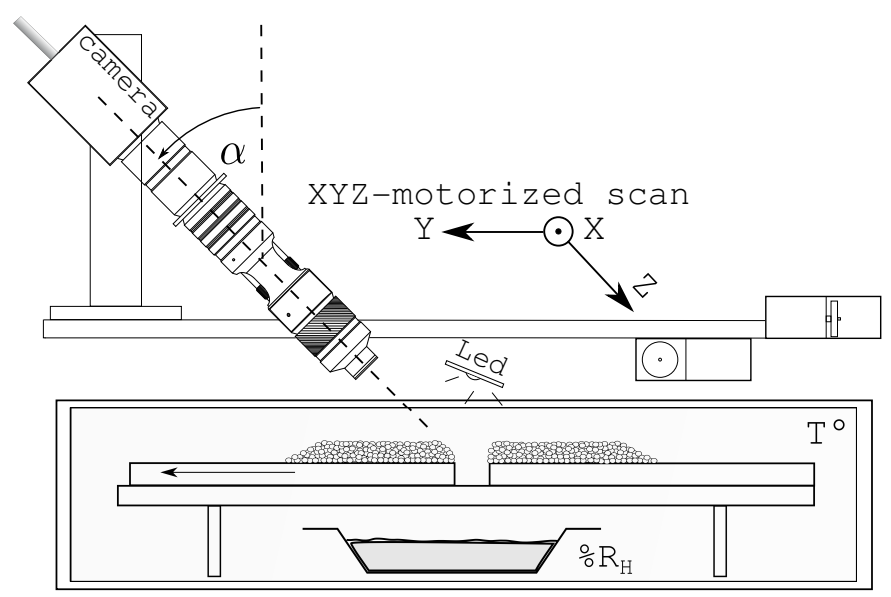

Fig. 1: Sketch of the experimental device designed to observe the ridge of a, nominally rectilinear, fracture produced in a thin layer of cohesive grains. The cohesion due to the formation of liquid bridges that form at the contact points between the grains is tuned by changing the relative humidity $R_{H}$ of the surrounding atmosphere. A CDD camera equipped with lenses, can be displaced along the fracture $(x$-axis) in order to obtain high resolution images of the ridge. The optical axis ( $z$-axis) is tilted to observe the grains of the ridge, only.

The granular material consists of spherical glass beads (Wheelabrator, sodosilicate glass), sieved in order to achieve rather monodisperse samples, which allows to discriminate the role played by the grain size. We report experiments for grain radius, $r=(90 \pm 10),(50 \pm 10)$, $(20 \pm 10) \mu \mathrm{m}$. Previous to the experiment, the various batches are maintained in a humid atmosphere, at $R_{H}=33 \%$, for days. The initial granular layer is obtained by spreading grains onto the upper surface of the plates that are initially in contact. An homogenous thickness $h$ (from 1 to $3 \mathrm{~mm}$ ) is achieved by levelling the free surface, using spacers. Once the layer is prepared, the chamber is closed and we permit the exchange of water between the granular layer and the surrounding atmosphere for $24 \mathrm{~h}$. We checked, waiting from 1.5 to $72 \mathrm{~h}$, that the results presented below are reproducible, to within the experimental precision, after 1 day.

The layer is broken by gently moving the two plates apart. Some grains fall in the gap, leaving on both sides two inclined walls whose roughness depends on the experimental conditions. The experiment relies in the analysis of the profile of the ridge at the free surface.

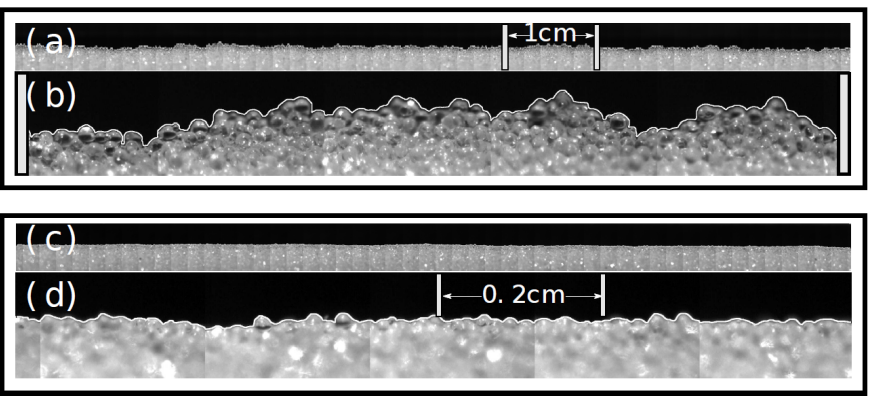

Fig. 2: Ridge images - (a) and (c) : large-scale images of the ridge are obtained by gathering a series of high-resolution pictures taken at various positions along the $x$-axis. (b) and (d) : detail of reconstructed images: the spatial resolution is enough to determine the profile of the ridge (white continuous line) at a scale much smaller than the grain size $[r=(90 \pm 10) \mu \mathrm{m}$, $h=3 \mathrm{~mm}$. (a)-(b): $R_{H}=100 \%$; (c)-(d): $\left.R_{H}=33 \%\right]$.

Special care has been taken in the design of the imaging technique (Fig. 1, top). Pictures of the ridge, from top, are obtained thanks to a digital video camera (DALSA, Falcon 1.4M100 HG) and a set of lenses (Thorlabs, MVL6.5X12Z and MVL6X05L) that achieve a resolution of $7.4 \times 7.4$ $\mu \mathrm{m}^{2}$ with a working distance of about $5.1 \mathrm{~cm}$. In these conditions, the field of view is only of $(1.04 \times 0.76) \mathrm{cm}^{2}$. In order to get a large-scale image (Fig. 2), the system automatically takes a series of pictures along the fracture ( $x$-axis). The camera is displaced by steps with a translation stage (Thorlabs, LTS300/M). The steps $(0.8 \mathrm{~cm})$ are chosen smaller that the width of the field of view $(1.04 \mathrm{~cm})$ so that successive pictures overlap. A $15 \mathrm{~cm}$-long image of the fracture is then reconstructed by adjusting their position by correlation of the parts they have in common.

The system is focused on the profile of the ridge, i.e. the sharp edge of the fracture at the free surface of the layer. In order to avoid imaging the grains that are below the free surface, along the inclined walls, the camera is tilted such that the optical axis makes the angle $\alpha$ (45 deg.) with the vertical. The camera can also be displaced in the transverse direction ( $y$-axis) and along the optical axis ( $z$ axis), which makes possible, if necessary, to respectively center the image of the ridge in the transverse direction and adjust the focus.

To sum up, the experimental device is designed to produce a fracture in a thin layer (thickness, $h$ ) of granular material (glass beads of typical radius $r$ ) in contact with a humid atmosphere (relative humidity, $R_{H}$ ). The observation system gives a $15 \mathrm{~cm}$-long image of the ridge at the free surface, with a resolution of $7.4 \mu \mathrm{m}$ in both the longitudinal ( $x$-axis) and transverse ( $y$-axis) directions.

Experimental results. - We propose here to analyze the fracture profiles in order to get clues on the inter- 
nal texture of the material previous to rupture.

Fracture profile. The first step of the analysis consists in extracting the ridge profile. The good contrast in the images allows to obtain easily the contour of the grains of the ridge (Fig. 2). Several profiles, i.e. the transverse position of the ridge $y$ as a function of the longitudinal position $x$, are displayed in Fig. 3. We report data obtained for the same grain radius, $r$, at various relative humidity $R_{H}$. One can immediately notice that the geometrical characteristics of the profile depend on the experimental conditions, here the width of the interface increases with the relative humidity $R_{H}$.

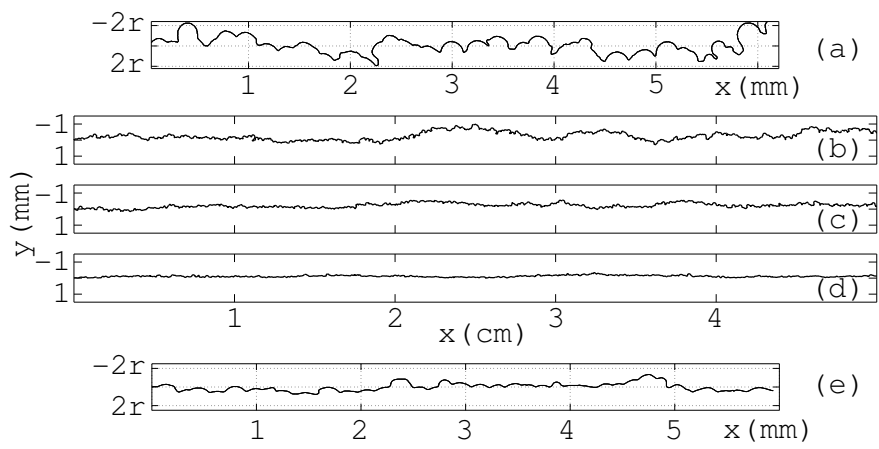

Fig. 3: Ridge profile at various relative humidity $R_{H}$. (b),(c) and (d), profiles of the fracture along $5 \mathrm{~cm}$ in the longitudinal direction. (a) and (e), enlargements of the profiles reported in (b) and (d), respectively. Note that, in the enlargments, one can clearly distinguish the grains $[h=3 \mathrm{~mm}, r=(90 \pm 10) \mu \mathrm{m}$. (a)-(b): $R_{H}=100 \%$; (c): $R_{H}=75 \%$; (d)-(e): $\left.R_{H}=33 \%\right]$.

Before discussing our analysis and results, we must comment on the physical origin of the transverse excursions of the ridge, and on the relevant length scales. We are interested in the internal structure of the material and, especially, in the size of the clusters that are likely to form in the material due to the cohesion associated with humidity. We already have clues that, if clusters form, they involve a small number of grains. Their typical size should not exceed 10 grain radius $[5,7]$.

However, in Fig. 3, we observe large transverse excursions [see for instance in (b), for $x$ between 2 and $3 \mathrm{~cm}$ ] irrelevant for the present study. Indeed, due to the way we produce the fracture, its profile is strictly linear in the lower plane of the granular layer, only. The upward propagation in the thickness, toward the free surface, is such that the transverse position of the ridge is imposed to within $\delta x \sim 2 h \delta \theta_{a}$, where $\delta \theta_{a}$ is the range of angle insuring the stability of the wall (in fact from the horizontal to the angle of avalanche $\theta_{a}$ ). Thus, $\delta \theta_{a}$ is typically of the order of unity, such that $\delta x$ can be large, of the order of the thickness. In order to remove any undesired effect associated to those excursions at large scale, we filter excessively slow variations in the longitudinal direction, i.e. along the $x$-axis. Expecting inhomogeneities of the internal texture of the material to a scale of at most 10 grains, we filter any excursion at a scale larger than 50 times the largest radius $r$ used in the experiments.

Finally, we mention that, for large cohesion, the profile sometimes exhibits overhangs (Fig. 3a). In this case, more than one transverse position $y$ can be associated to the longitudinal position $x$. Some analysis techniques used in the following do not permit such event. To avoid multivaluated transverse position $y$, we thus arbitrarily associate to $x$ the maximum value of $y$. Such procedure does not alter the conclusion of the study as overhangs remain rare.
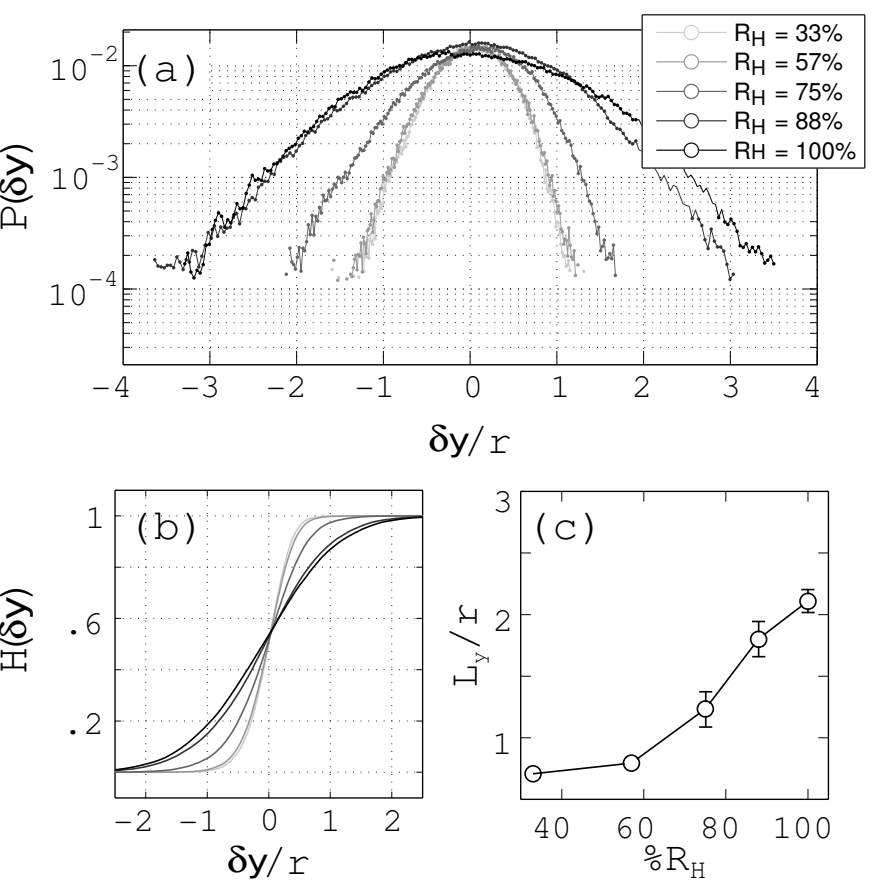

Fig. 4: (a) Probability distribution $P(\delta y)$ of the transverse displacement $\delta y$ for various relative humidity $R_{H}$. We observe a clear widening of the distribution when $R_{H}$ is increased. (b) Corresponding cumulative probability $H(\delta y)$. (c) Width $L_{y}$ vs. relative humidity $R_{H}[h=3 \mathrm{~mm}$ and $r=(90 \pm 10) \mu \mathrm{m}]$.

Typical transverse scale. First, of all, we extract the width, $L_{y}$, of the ridge profile in the transverse direction. In order to measure $L_{y}$, we define $\delta y \equiv y-\langle y\rangle$, the displacement of the transverse position from its average $\bar{y}$. In Fig. 4, we report typical probability distributions $P(\delta y)$ of $\delta y$, taking into account values measured all along the ridge profile. We observe a clear widening of the distributions $P(\delta y)$ for increasing values of the relative humidity $R_{H}$. The transverse scale (width) of the profile can be characterized by reporting the mean square displacement $L_{y} \equiv \sqrt{\left\langle\Delta y^{2}\right\rangle}$ or, equivalently by displaying the cumulative probability $H(\delta y) \equiv \int_{\infty}^{\delta y} P\left(\delta y^{\prime}\right) d\left(\delta y^{\prime}\right)$.

The measurements reported in Fig. 4c reveal that the width of the profile, $L_{y}$, which remains of the order of the grain size, is typically multiplied by a factor 3 when the relative humidity is increased from $R_{H}<33 \%$ (dry atmosphere) to $R_{H}=100 \%$ (saturated atmosphere). 
Typical longitudinal scale. We observed above that the width of the ridge increases with the relative humidity $R_{H}$. However, the feature is not sufficient to reveal the formation of clusters in the bulk of the material. In that case, one should observe the appearance of a characteristic length of the same order in the longitudinal direction too.
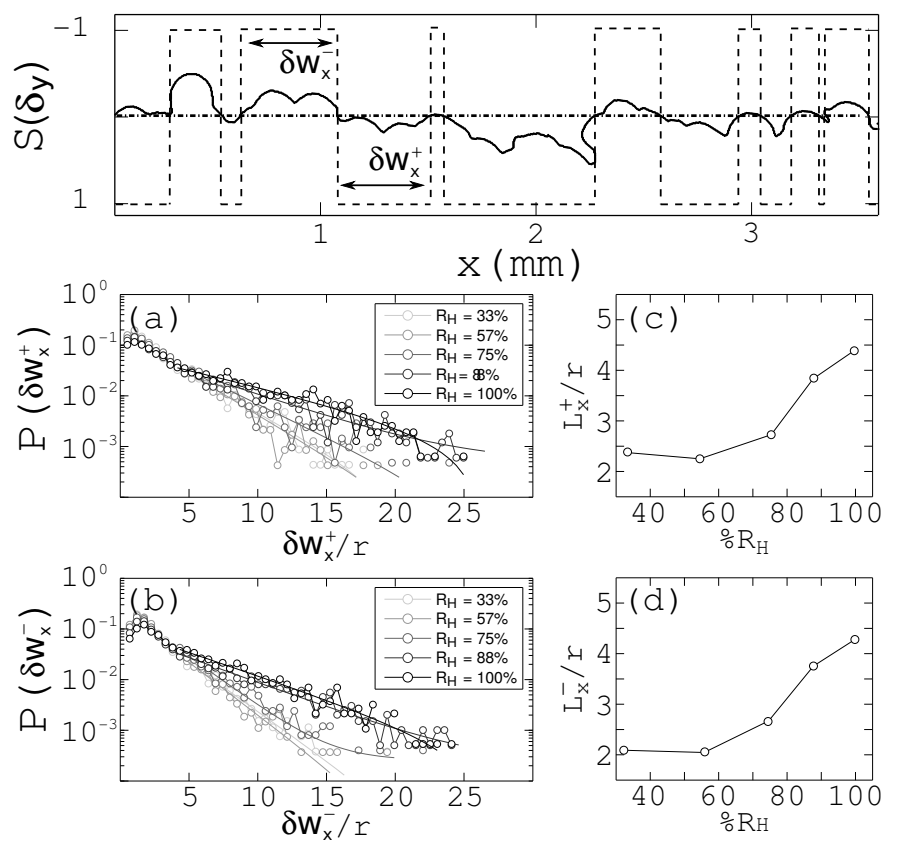

Fig. 5: Top: Typical binarized profile $S(\delta y)$ vs. $x$ - Continuous line: $\delta y$ vs. $x$, dashed line: $S(\delta y)$ vs. $x$. From $S(\delta y)$, we extract the length $\delta w_{x}^{ \pm}$of the segments in which $\delta y$ keeps the same sign. (a) and (b): Probability distributions $P\left(\delta_{x}^{+}\right)$and $P\left(\delta_{x}^{-}\right)$ (Points: experimental data - Lines: guide to the eye). (c) and (d): Corresponding characteristic length, $L_{x}^{ \pm}$as function of the relative humidity $R_{H}[h=3 \mathrm{~mm}$ and $r=(90 \pm 10) \mu \mathrm{m}]$.

One convenient way to reveal a characteristic length in the longitudinal direction consists in assessing the spatial extension, along the $x$-axis, of the excursions of the ridge $y$ around its average position $\langle y\rangle$. We consider binarized profile $S(\delta y)$, where $S$ is the sign function (Fig. 5, top). From $S(\delta y)$, we extract the length $\delta w_{x}^{ \pm}$of the segments in which $\delta y$ keeps the same sign, negative $\left(^{-}\right)$or positive $\left(^{+}\right)$. Then, we consider the probability distribution $P\left(\delta w_{x}^{ \pm}\right)$of the width $\delta w_{x}^{ \pm}$. From the data, we obtain two measures, $L_{x}^{ \pm} \equiv\left\langle\delta w_{x}^{ \pm}\right\rangle$, of the characteristic size in the longitudinal direction [Fig. 5, (c)-(d)]. We observe, even if positive and negative excursions are not strictly symmetric, that the lengths $L_{x}^{+}$and $L_{x}^{-}$are almost equal $L_{x}^{+} \simeq L_{x}^{-} \equiv L_{x}$. The length, $L_{x}$, which also remains of the order of the grain size, increases with the relative humidity $R_{H}$.

Clusters. The profile exhibits two typical length scales, $L_{x}$ and $L_{y}$, respectively in the longitudinal and the transverse directions (Fig. 6a). We observe that $L_{x}$ and $L_{y}$ are strongly correlated, $L_{x}$ being almost twice $L_{y}$.
Note that this factor originates, at least partly, from the difference in the analysis techniques used for the transverse and longitudinal directions. These data thus reveal that the ridge, and thus the fracture propagates in a material made of units having the same characteristic size $L\left(\equiv L_{y}\right.$, arbitrarily) in the longitudinal and transverse directions. This experimental feature is qualitatively compatible with the existence of cohesive clusters of size $L$ in the bulk of the material.
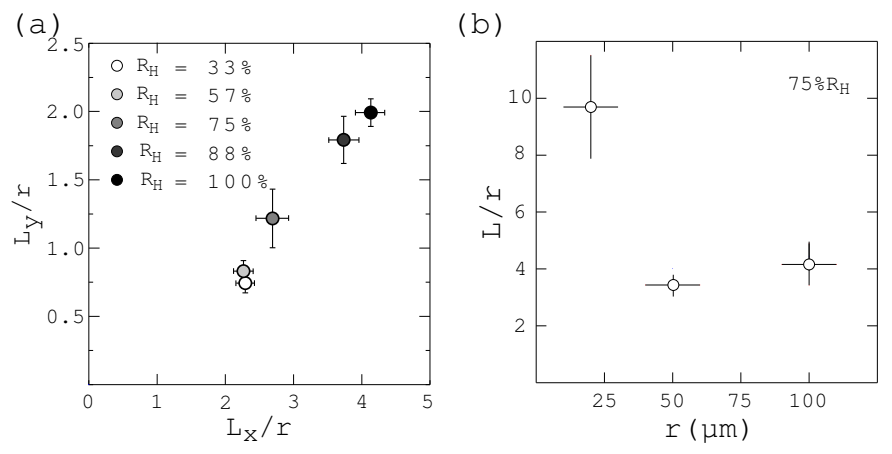

Fig. 6: (a) Longitudinal characteristic length $L_{x}$ vs. transverse characteristic length $L_{y}$. (b): Characteristic length $L$ vs. grain radius $r$ for a fixed relative humidity $R_{H}=75 \%$.

The lengthscale increases with relative humidity $R_{H}$. Quantitatively, one expects the typical size of the clusters to tend to the grain size in the limit of small humidity [7]. Accordingly, we observe in Fig. 6 a that $L$ is of the order of the grain radius $r$ for the smallest values of $R_{H}(<50 \%$ typically, Fig. 4). In the opposite limit, for a saturated atmosphere $\left(R_{H}=100 \%\right), L$ reaches a maximum value of about a few (i.e. 4$)$ grain radius, again in accordance with previous estimates $[5,7]$.

The cluster size, in terms of number of grains, does not depend on $r$ for the larger grains $[r>50 \mu \mathrm{m}$, typically. For smaller grains, other sources of cohesion (Van der Waals forces, for instance) become significant and the conclusion does not hold]. This striking independence of $L / r$ on the grain size is a strong result that explains the dependency of fracture patterns in cohesive granular matter on grain size and humidity [7]. Alarcón et al proposed that $L / r \simeq 1+$ $\sigma_{s} r^{2} / \beta \xi$ where $\xi$ is the typical size of the asperities at the grain surface, $\beta$ an energy scale and $\sigma_{s}$ the tensile stress due to the liquid bridges. For small humidity content, the bridges form around the asperities only, and $\sigma_{s} \propto 1 / r^{2}$, which results in the indepence of $L / r$ on $r$. Our results are in agreement with these former observations.

Self-affine scaling. In order to get further insight into the morphology of the ridge profile, we study the properties of the increments $\Delta y$ and their correlation through the longitudinal coordinate $x$. We consider the variation $\Delta y$ of the transverse position $y$ over a length $\delta x$ along the ridge, i.e. $\Delta y(x, \delta x) \equiv y(x+\delta x)-y(x)$. More specifically, we study the scaling behavior of the root mean square (rms) of the height fluctuations $\sigma_{\Delta y}(\delta x)=\left\langle\Delta y^{2}\right\rangle^{1 / 2}$ as a 


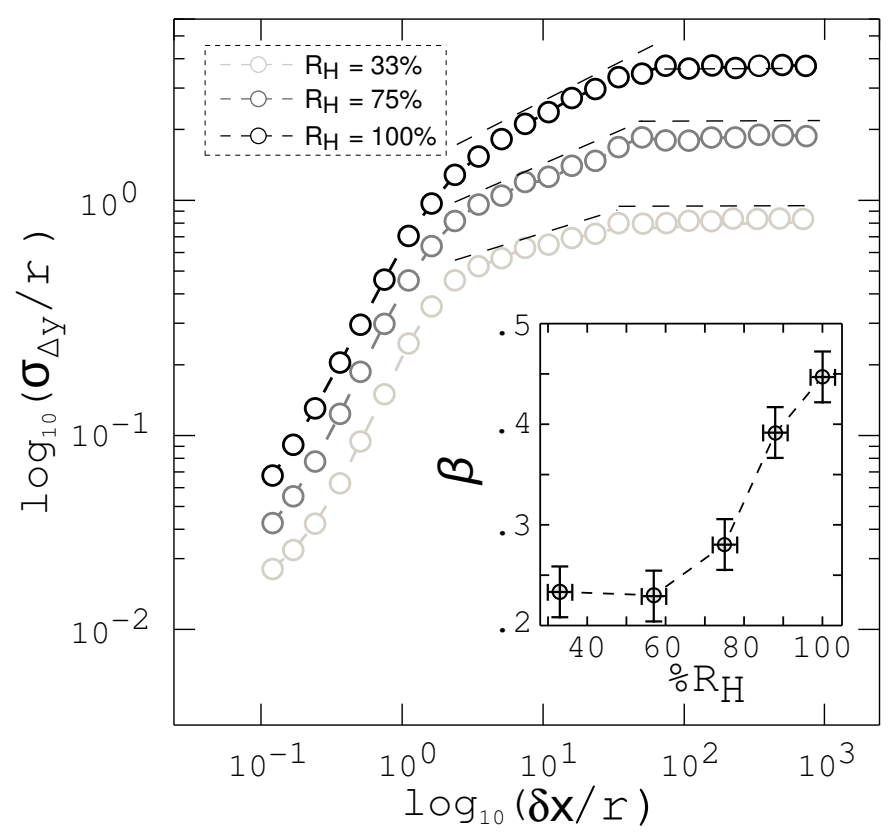

Fig. 7: Mean square displacement $\sigma_{\Delta y}$ vs. $\delta x-$ Inset: Slope $\beta$ vs. relative humidity $R_{H}[h=3 \mathrm{~mm}$ and $r=(90 \pm 10) \mu \mathrm{m}]$.

function of the longitudinal length scale $\delta x$. Such analysis is classically used and developed in the fractography community to reveal spatial correlations along crack interfaces. Indeed, since the pioneering work that evidenced the selfaffine character of fracture surfaces of metals [10], a considerable amount of experimental and theoretical works (see the recent review [11] and references therein) have studied the statistical scale invariance properties of crack surfaces and crack lines $y(x)$. Figure 7 displays the scaling behavior of the root mean square (rms) of the crack height fluctuations $\sigma_{\Delta y}(\delta x)$ for different humidity rates $R_{H}$. We can distinguish three different regimes:

At small scales, below the grain size, $\delta x<2 r$, the various curves $\sigma_{\Delta y}$ almost superpose since the fluctuations of the interface are dominated by the shape of the grains. However, we notice a significant shift towards larger transversal excursions when the relative humidity is increased: $\sigma_{\Delta y}$ is larger at a given $\delta x$ for larger $R_{H}$. This result is compatible with the observation, in Figs. 2(b),(d) and $3(\mathrm{a}),(\mathrm{d})$, of individual grains that can remain stable more and more ahead from the interface when $R_{H}$ is increased.

In the opposite limit, at the largest scales, $\delta x \rightarrow \infty, \sigma_{\Delta y}$ tends to a constant value which corresponds to the typical transverse width of the crack interface, $L_{y}$, reported in a previous section. As previously observed, this typical transverse width increases with the humidity rate $R_{H}$. Nevertheless, it is important to be aware that this value can be affected by the filtering procedure of the profiles.

Let us now focus on the intermediate regime. Interestingly, we can observe a self-affine scaling behavior of the fracture profile, in an intermediate range of scales: the rms of the height fluctuations $\sigma_{\Delta y}$ systematically increases with the size of the measuring window $\delta x$ according to $\sigma_{\Delta y} \propto \delta x^{\beta}$, with the scaling exponent $\beta$ - usually called roughness exponent in the fractography literature [10-12]. In the inset of Fig. 7 , we observe that this exponent $\beta$ increases with $R_{H}$, from $\beta=0.24 \pm 0.05$ when the relative humidity is around $R_{H} \simeq 40 \%$ up to $\beta=0.45 \pm 0.05$ when $R_{H}=100 \%$. One can notice that such evolution of the roughness exponent $\beta$ with the humidity rate is similar to the one of the width of the statistical distribution $P(\delta y)$ of the transverse displacement of the crack front, shown in Fig. 4. This self-affine scaling behavior observed on a decade shows that the crack front displays spatial correlations up to $\delta x^{*} \simeq 50 \mathrm{r}$. However, this upper characteristic correlation scale seems independent of $R_{H}$, and thus is probably fixed by the filtering procedure of the profile.

The fracture in our cohesive granular layer should follow inter-clusters path of minimum strength. Thus, the increase of the scaling exponent $\beta$ with the relative humidity $R_{H}$ appears consistent with the formation of cohesive clusters, which sizes increase with $R_{H}$, as evidenced in the previous section. Finally, the roughness exponent (in particular, its evolution as function of $R_{H}$, i.e. of the cohesive clusters size) appears in remarkable agreement with values reported recently for fracture surfaces in sintered materials and, moreover, with their dependency on the porosity of those samples [13].

Conclusion. - We analyzed in details the profile of the fracture ridge in cohesive granular matter. The profile reveals a unique underlying length scale, $L$, which, proportional to the grain size, is larger for larger humidity content. Upon condensation in a granular material in contact with a humid atmosphere, water is not homogeneously distributed. The material is thus more cohesive in regions of typical size $L$, the clusters, where the water content is larger. The results are particularly important as such underlying structures govern the mechanical properties of the material at large scales.

This work was funded by the CONICYT POSTDOCTORADO-2013 74140057 and from Région Rhône-Alpes CMIRA Pro 14-0043385.

\section{REFERENCES}

[1] Hornbaker D. J. , Albert R., Albert I., Barabási A.-L. AND Schiffer P., Nature, 387 (1997) 765.

[2] Samadani A. and Kudrolli A., Phys. Rev. E, 64 (2001) 050301.

[3] Bocquet L., Charlaix E., Ciliberto S. and Crassous J., Nature, London 396 (1998) 735.

[4] Losert W., Géminard J.-C., Nasuno S. and Gollub J. P., Phys. Rev. E, 61 (2000) 4060.

[5] Freyssingeas E., Dalbe M.-J. and Géminard J.-C., Phys. Rev. E, 83 (2011) 051307. 
[6] Alarcón H., Ramos O., Vanel L., Vittoz F., Melo F. And GÉminard J.-C., Phys. Rev. Lett., 105 (2010) 208001.

[7] Alarcón H., Géminard J.-C. and Melo F., Phys. Rev. E, 86 (2012) 061303.

[8] Scheel M., Seemann R., Brinkmann M., Di Michiel M., Sheppard A., Breidenbach B. and Herminghaus S., Nature, 7 (2008) 189-193.

[9] Rockland L. B., Anal. Chem., 32 (1960) 10.

[10] Mandelbrot B. B., Passoja D. E. and Paullay A. J., Nature, 308 (1984) 721.

[11] Bonamy D. and Bouchaud E., Phys. Rep., 498 (2011) 1.

[12] Santucci S., Måløy K.J., Delaplace A., Mathiesen J., Hansen A., Bakke J. O. H., Schmittbuhl J., Vanel L. And Ray P., Phys. Rev. E, 75 (2007) 016104.

[13] Cambonie T., Bares J., Hattali M. L., Bonamy D., Lazarus V. and Auradou H., Phys. Rev. E, 91 (2015) 012406. 